\title{
Influence De L'exploitation Artisanale Du Sable Sur La Santé Et La Sécurité Des Artisans Et L'environnement: Cas De La Carrière De Nkol'Ossananga, Région Du Centre Cameroun
}

\author{
Clautaire Mwebi Ekengoue \\ Popular Outcome for Fundamental Sciences and Innovative \\ (SIPOF), Yaoundé, Cameroun \\ Laboratoire de Géologie de l'Environnement, Département des Sciences de \\ la Terre, Université de Dschang, Ouest-Cameroun \\ Rodrigue Fotie Lele \\ Green Heart International (GHI), Headquarter Immeuble Waffo Pierre, Street \\ Afrique Construction Ndiandam, BP 1450 Bafoussam Cameroun

\section{Armand Kagou Dongmo} \\ Laboratoire de Géologie de l'Environnement, Département des Sciences de \\ la Terre, Université de Dschang, Ouest-Cameroun
}

Doi: 10.19044/esj.2018.v14n15p246 URL:http://dx.doi.org/10.19044/esj.2018.v14n15p246

\begin{abstract}
Sand is classified among the low value development minerals, even though it is massively used in the world. The exploitation of that construction mineral at Nkol'Ossananga, around Yaounde, is done by craftsmen in unsafe conditions, such as: the exposure to a wide range of diseases and the destruction of the environment. We carried out a study on occupational health and safety and environmental impacts on the Nkol'ossananga site. Our method consisted firstly of the bibliographic review, sandpit quarry investigations afterwards, and lastly the data analysis. Tabulation of results showed that the population in the site is essentially threatened by diseases such as dysentery (15\%), fibromyalgia (25\%), typhoid fever (55\%), otalgia (10\%), ocular pains $(15 \%)$, and malaria (45\%). Craftmen work without any personal protective equipment (PPE) and are often victims of drowning and bacterial infections. The high demand of sand in Yaoundé involves the development of the activity in other sites and, in consequence, there is a deforestation and disappearance of many animals and vegetables species. The soil and water pollution is also noticeable due to pouring of engines waste oil in the site.
\end{abstract}


Keywords: Yaoundé, Artisanal Sand Exploitation, Health and Safety, Environment

\section{Résumé}

Le sable est classé parmi les minéraux de développement de faible valeur, pourtant fortement utilisé dans le monde pour les travaux de construction d'ouvrages d'art. L'exploitation de ce matériel de construction s'effectue essentiellement de façon rudimentaire par des artisans qui courent ainsi de nombreux risques, notamment l'exposition à de nombreuses maladies et la dégradation de l'environnement. Nous avons mené une étude sur l'influence de l'exploitation artisanale du sable sur l'environnement, la santé et la sécurité des artisans dans la carrière de Nkol'Ossananga, Région du Centre Cameroun. La démarche méthodologique utilisée a consisté d'abord en la consultation des documents, ensuite aux investigations sur le terrain et enfin au traitement des données et à l'analyse des résultats. Le dépouillement des résultats de l'enquête ouverte auprès des artisans à Nkol'Ossananga a montré que la population rencontrée sur le site est menacée par des maladies telles que la dysenterie (15\%), la fibromyalgie (25\%), la fièvre typhoïde $(55 \%)$, l'otalgie (10\%), les douleurs oculaires (15\%) et le paludisme (45\%). Les artisans de Nkol'Ossananga travaillent sans équipements de protection individuelle (EPI) et sont souvent victimes des noyades et des infections bactériennes. La forte exploitation du sable dans la région entraîne la recherche de nouveaux sites, d'où la destruction des espaces de forêts et en conséquence la déforestation et la disparition de nombreuses espèces animales et végétales. La pollution des sols et des eaux est également sensible suite au déversement des huiles usées d'engins sur le site.

Mots-clés : Yaoundé, Exploitation Artisanale du Sable, Santé-Sécurité, Environnement

\section{Introduction}

L'exploitation artisanale des minéraux de développement de faible valeur en général et celle des matériaux de construction en particulier est devenue une réalité incontournable, une occupation économique au même titre que l'agriculture et l'élevage, et génère des devises pour le compte de l'économie nationale (Sba-Ecosy-CEDRES, 2011). Au Cameroun, et plus particulièrement dans la région du Centre, cette exploitation est réduite à l'extraction du sable dans le lit du fleuve Sanaga. Malgré la prospérité financière que cette activité offre aux populations concernées, elle comporte à l'origine des dangers considérables, en ce qui concerne la santé et la sécurité des artisans mais aussi des impacts environnementaux énormes. Pour les 
populations, les effets de l'exploitation minière artisanale ont plusieurs visages (Mutsima et al., 2015): poussières, remblaies, présence des mineurs dans les sites (Kyamwami, 2013 ; Razack, 2012), manque d'eau potable, transfert démographique du milieu rural vers les mines (Sinonda, 2010 ; Razack, 2012), insécurité liée au trafic d'armes (Geenen et al., 2010), origine du lien existant entre ressources naturelles et conflits remarqué par Collier (2000), Razack (2012) et Billon (2001). L'exploitation minière artisanale est, dans la plupart des cas, la source de nombreux accidents de travail le plus souvent mortels (Razack, 2012). Sur les sites d'exploitation, on note l'absence des règles élémentaires d'hygiène et de sécurité caractérisée par la précarité des conditions de travail (absence des équipements de protection individuelle, etc.) exposant ainsi les artisans à de nombreuses maladies telles que les infections pulmonaires liées aux poussières, les risques de blessures dues aux éclats de pierres pour le cas des carrières de pierres, les risques de surdité liés aux bruits permanents dans le site et les affections oculaires (Goh, 2016). En ce qui concerne l'environnement, les effets négatifs liés à l'activité minière artisanale varient le plus souvent en fonction des contextes géographiques, des substances exploitées ainsi que du matériel et des méthodes d'exploitation employées (GADHOP, 2012). Parmi ces impacts, on peut citer: la destruction de la faune et de la flore (Goh, 2016 ; Midende, 2010 ; Kemgang, 2015), la destruction du sol, la pollution des eaux et de l'air (Razack, 2012 ; GADHOP, 2012), l'augmentation de la turbidité des eaux dans lesquelles l'exploitation est effectuée, l'insalubrité provenant de l'accumulation des déchets organiques et autres accessoires utilisés dans le site.

Depuis ces dernières années, du fait de la forte demande en sable, l'exploitation de ce matériau dans le lit du fleuve Sanaga s'est intensifiée, occupant une population de plus en plus importante. Cette étude vise à évaluer l'influence de l'activité d'exploitation artisanale du sable sur l'environnement, la santé et la sécurité des artisans dans la carrière du sable de Nkol'Ossananga, Région du Centre Cameroun.

L'article est structuré de la manière suivante : dans la deuxième partie, nous avons mis en évidence la méthode de travail et le matériel employés, aussi bien que la carte de localisation du site d'étude. En ce qui concerne la troisième et la quatrième partie, les résultats obtenus durant toute l'étude sont présentés et discutés. Comme conclusion de ce travail, nous présentons la ligne à suivre pour améliorer l'état de santé et de sécurité des artisans ainsi que la préservation de l'environnement.

\section{Matériel et méthode}

Pour atteindre les objectifs visés, l'ensemble du matériel utilisé pendant notre étude est constitué d'un appareil photo numérique, du GPS, des fiches d'enquête et d'un ordinateur portable. La démarche méthodologique 
mise en place a été constituée de trois étapes: d'abord la recherche bibliographique, ensuite les investigations sur le terrain (les enquêtes auprès des artisans et la prise des coordonnées de la zone d'étude, etc.) et finalement le traitement et l'analyse des données : dépouillement des résultats d'enquête et cartographie du site (Figure 1).

L'étape portant sur la recherche bibliographique a consisté en la collecte des informations sur les impacts sociaux et environnementaux de l'exploitation des carrières artisanales de sable dans le monde en général et au Cameroun en particulier. Cette phase de collecte de données nous a permis d'avoir une idée claire sur la méthode d'exploitation du sable marin, et plus précisément celui du fleuve Sanaga dans la région du Centre Cameroun, avant notre descente sur le terrain. Elle nous a également permis de connaître à priori le matériel utilisé par les artisans dans l'exploitation artisanale du sable marin. Globalement, il s'agit de la phase qui nous a permit de faire quelques prévisions sur les résultats attendues. Elle a facilité la conception de la fiche d'enquête ainsi que la discussion des différents résultats obtenus après analyse des données obtenues pendant les travaux de terrain.

Les travaux de terrain ont été constitués essentiellement de la collecte des données nécessaires pour la réalisation effective de l'étude. La collecte des données a été faite d'abord à partir de l'observation, ensuite des enquêtes auprès des artisans et enfin à partir de la prise des coordonnées GPS pour la localisation du site.

* Observations : ici, il a été question d'identifier le matériel utilisé par les artisans dans l'exploitation artisanale du sable Sanaga, les techniques d'exploitation du sable Sanaga qu'ils emploient, les différents risques liés à l'exploitation auxquels les artisans sont exposés ainsi que les aliments consommés au quotidien par les artisans sur le site pendant l'activité d'exploitation.

* Enquêtes auprès des artisans : pour cette phase, un questionnaire a été conçu, nous permettant de collecter les informations essentielles sur les différentes maladies dont souffrent les artisans depuis leur arrivé sur le site, la quantité de sable pouvant être ramassée au quotidien par chaque plongeur, les revenus journaliers de chaque classe d'artisans présente sur le site, le statut matrimonial des artisans rencontrés sur le site, leur niveau de scolarisation, leur niveau de connaissance des dangers qu'ils courent pendant l'exploitation du sable (surtout les plongeurs). Ici, il a été également question pour nous d'avoir une idée claire sur la quantité de bois nécessaire pour la fabrication d'une pirogue, la provenance et la quantité de planches qu'un arbre de la zone peut produire.

* Localisation du site : quant à la localisation du site, nous nous sommes servis d'un GPS afin de collecter les coordonnées sur différents points 
du site. Ces coordonnées doivent être traitées sur un logiciel afin de produire une carte de localisation du site appropriée.

Dans la phase portant sur les enquêtes auprès des artisans, nous avons utilisé la méthode semi-participative avec des entretiens structurés et une fiche d'enquête élaborée. Nos questions orales ou mentionnées sur les fiches d'enquêtes, étaient de sorte à accorder beaucoup plus d'attention aux attitudes des artisans, leur apparence physique, leur état de santé et les risques auxquels ils sont exposés pendant l'activité d'exploitation artisanale du sable Sanaga. Une trentaine d'artisans représentant le tiers de la population in-situ ont été enquêtés. Par ailleurs, grâce à l'analyse des techniques d'exploitation et le matériel utilisé, une attention particulière a été portée sur l'état de dégradation de l'environnement où l'exploitation est menée. Le niveau de dégradation de la qualité d'eau du fleuve Sanaga a été évalué à la suite du résultat d'analyse d'un échantillon de cette eau au laboratoire des sols et géologie de l'environnement de la Faculté d'Agronomie et des Sciences Agricoles (FASA) de l'Université de Dschang.

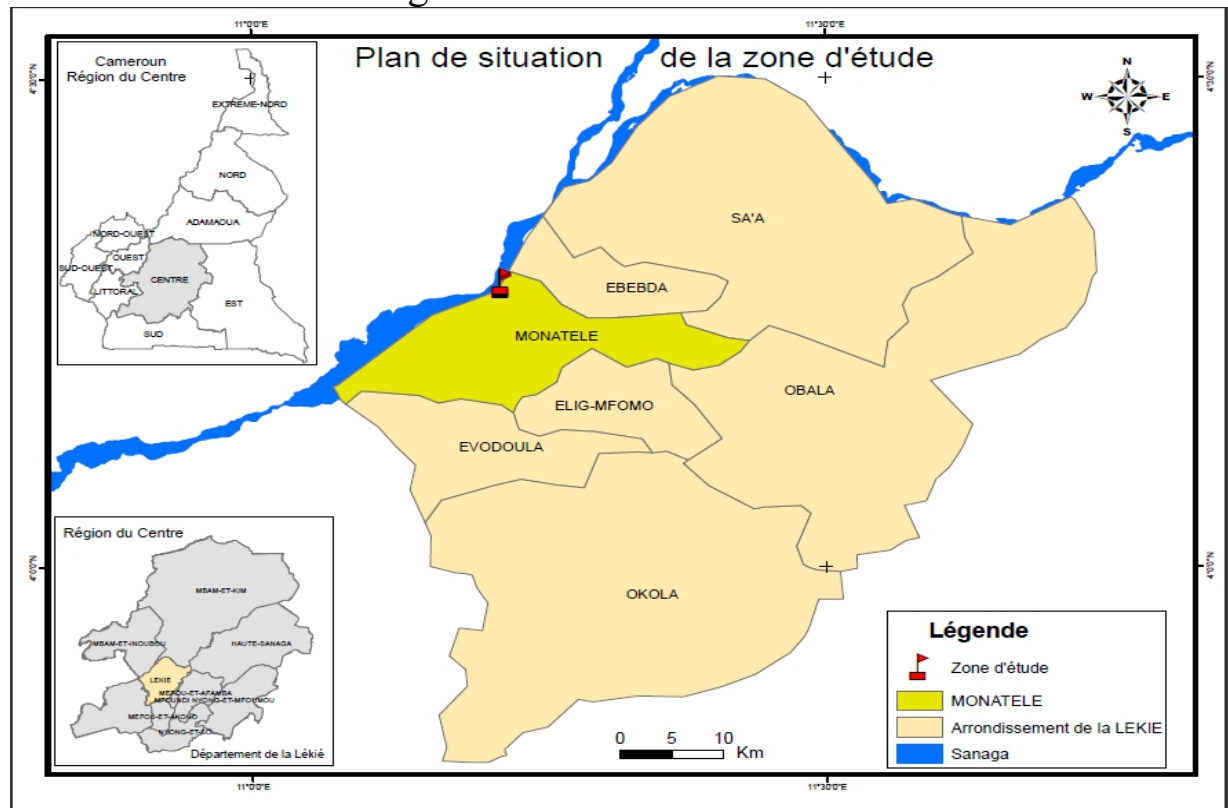

Figure 1. Carte de localisation de la zone d'étude. Source : travaux personnels.

\section{Résultats}

\section{Exploitation du sable à Nkol'Ossananga : organisation et étapes de} l'activité

L'exploitation du sable à Nkol'Ossananga est hiérarchiquement organisée de la manière suivante (Tableau I): 
Table 1. Organisation de l'activité d'exploitation artisanale du sable Sanaga à

Nkol'Ossananga

\begin{tabular}{|c|c|c|c|c|}
\hline Responsable & Contrôleur & Chefs de postes & Plongeurs & Chargeurs \\
\hline $\begin{array}{l}\text { Propriétaire du site } \\
\text { d'exploitation } \\
\text { Il joue le rôle } \\
\text { d'arbitre en cas de } \\
\text { litiges. } \\
\text { Il est assisté d'un } \\
\text { comptable chargé } \\
\text { d'évaluer ses } \\
\text { revenus journaliers } \\
\text { et d'un contrôleur. }\end{array}$ & $\begin{array}{l}\text { Il est chargé du } \\
\text { contrôle des } \\
\text { camions de } \\
\text { transport entrant } \\
\text { dans la carrière et } \\
\text { de la récupération } \\
\text { de la taxe à la } \\
\text { vente aux chefs } \\
\text { de postes }\end{array}$ & $\begin{array}{l}\text { Propriétaires d'un } \\
\text { espace bien défini } \\
\text { en bordure du } \\
\text { fleuve, réservé au } \\
\text { dépôt du sable } \\
\text { apporté par les } \\
\text { plongeurs. }\end{array}$ & $\begin{array}{c}\text { Chargés de la } \\
\text { collecte du } \\
\text { sable dans le } \\
\text { lit fluvial }\end{array}$ & $\begin{array}{l}\text { Chargés de vider } \\
\text { les pirogues } \\
\text { pleines du sable } \\
\text { apportées par les } \\
\text { plongeurs. } \\
\text { Ils s'occupent } \\
\text { aussi du } \\
\text { chargement des } \\
\text { camions de } \\
\text { transport arrivant } \\
\text { sur le site. }\end{array}$ \\
\hline
\end{tabular}

A côté de ce grand ensemble se rencontrent les commerçants, les menuisiers et les mécaniciens (Figure 2).

L'exploitation du sable à Nkol'Ossananga se fait en trois grandes étapes : le ramassage, le stockage et la vente. Le ramassage du sable est pratiqué par les plongeurs (Figure 3a). Ces derniers mènent leur activité de façon purement archaïque, en utilisant des seaux et des pirogues comme matériels de travail. Chez les plongeurs, cette activité consiste à se déplacer au moyen de leurs pirogues, de la rive jusqu'au milieu du fleuve, où ils font plusieurs plongées.

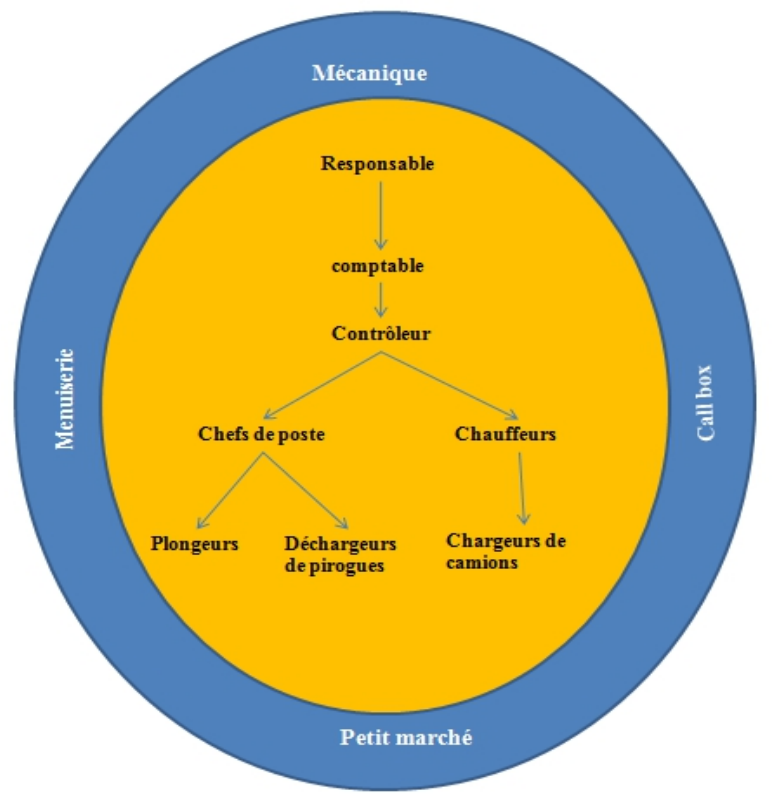

Figure 2. Organisation de la carrière artisanale de sable de Nkol'Ossananga

Ils remontent en surface avec du sable ramassé du lit fluviatile, qu'ils déchargent dans la pirogue. Généralement, ils travaillent pour les chefs de 
postes qui sont les propriétaires de pirogues. Très souvent, deux plongeurs s'associent et partent avec quatre (4) ou cinq (5) pirogues, malgré la forte turbidité du fleuve, pour augmenter leurs revenus journaliers. Déplacer plusieurs pirogues à 2, semble plus facile pour les plongeurs. En effet, au regard de la distance qui sépare le bord du fleuve du lieu de ramassage du sable, mais aussi, au regard des risques liés à la turbidité du fleuve Sanaga, les plongeurs préfèrent le plus souvent s'associer par deux et se déplacer avec 3, 4 voire 5 pirogues liées les unes aux autres par des ficelles rigides (voir Figure 3 ci-dessous) pour maximiser la quantité de sable à ramasser et ainsi éviter de faire plusieurs aller-retour.

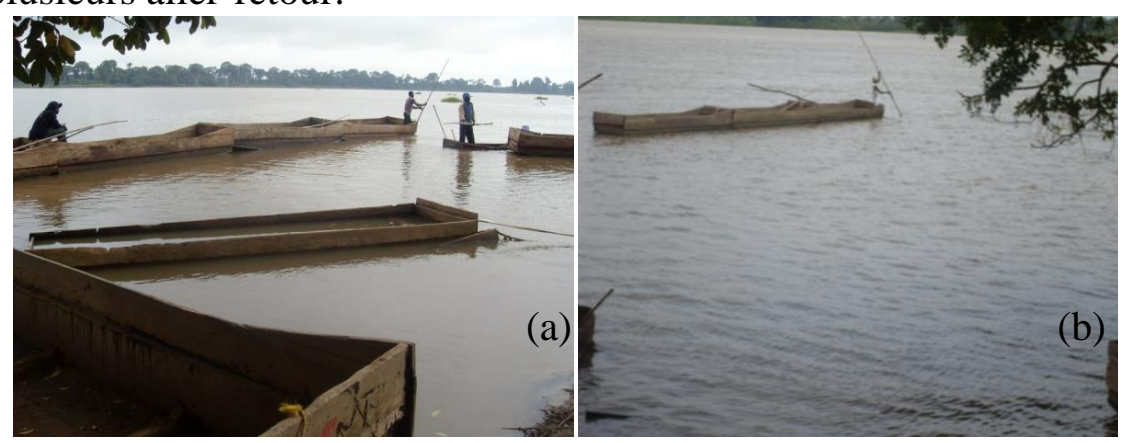

Figure 3. Deux plongeurs se déplaçant avec quatre (a) pirogues et un seul plongeur se déplaçant avec trois pirogues (b) liées les unes aux autres par des ficelles rigides.

Le stockage (Figure $4 \mathrm{~b}$ ) concerne les déchargeurs de pirogue. Il s'agit d'une classe d'artisans dont le rôle est de décharger à l'aide des pelles, les pirogues chargées du sable ramenées par les plongeurs. Le stockage du sable est une tâche qui, généralement, nécessite l'intervention de deux déchargeurs qui par permutations successives, vident la pirogue du sable. La commercialisation (Figure 4c) ou la vente des produits stockés varie en fonction de la demande de la clientèle. Cette activité met en jeu deux groupes d'individus : les camionneurs pour l'achat du sable et les chargeurs pour le remplissage des camions. Ce chargement se fait par huit (8) chargeurs qui se disputent généralement la place au bord des camions, en raison de 4 de chaque côté, laissant peu d'espace entre eux.

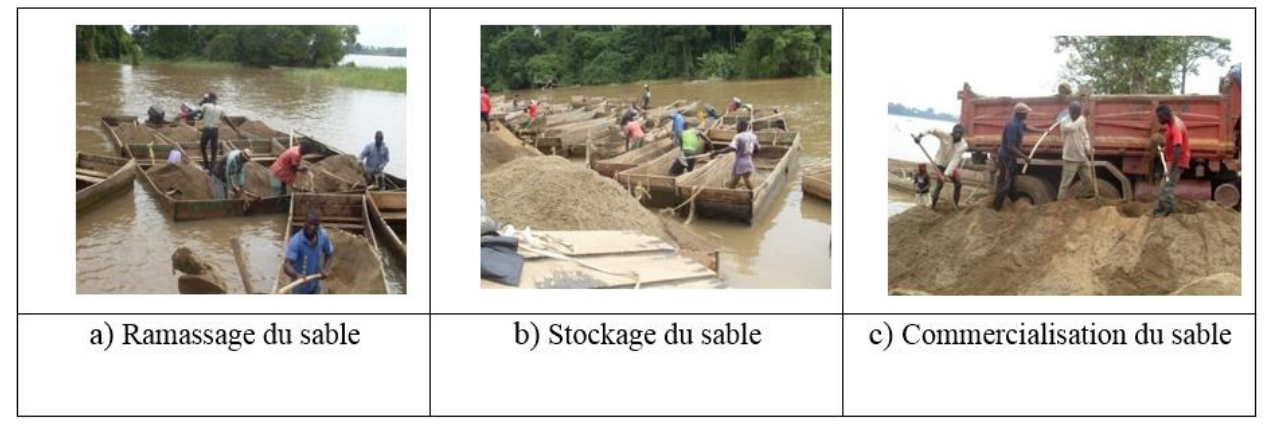

Figure 4. Etapes d'exploitation du sable à Nkol'ossananga. 


\section{Impacts de l'exploitation du sable sur la santé des artisans}

De façon générale, les aliments consommés dans la carrière de sable de Nkol'Ossananga ne sont pas variés. Il s'agit d'une gamme sélective, constituée essentiellement des céréales, de la matière grasse, des tubercules et de la boisson (cf. Tableau II). A Nkol'Ossananga, l'environnement de travail est inapproprié. L'absence d'eau potable et des bacs à ordures obligent les commerçantes à utiliser le peu d'eau dont elles disposent de façon rationnelle, mais aussi toute personne qui s'y trouve à jeter les ordures dans la sablière. Dans la carrière d'exploitation artisanale du sable de Nkol'Ossananga, on observe également l'absence des éléments de première nécessité dont la boîte à pharmacie, le dortoir, le restaurant, les ateliers de menuiserie pour la fabrication des pirogues et de mécanique pour le dépannage des camions et des autres véhicules entrant au site. Par ailleurs, de part et d'autre du site, on note la présence des marres d'eaux stagnantes, lieux de prolifération de moustiques. Les résultats de l'enquête auprès des artisans à Nkol'Ossananga sur leur état de santé ont montré que ces derniers sont plus menacés par la fièvre typhoïde et le paludisme (Figure 5).

Tableau II. Aliments consommés au quotidien par les artisans de la carrière d'exploitation artisanale du sable de Nkol'Ossananga.

\begin{tabular}{cc}
\hline Classe d'aliments & Aliments \\
\hline Matières grasses & Beurre, huile de palme, \\
Céréales & Haricot, riz, pain, beignets, maïs. \\
Viande/poisons & Viande de Boeuf et poisson \\
Boissons & Bière, jus (coca-cola,..), vin de Palme \\
Légumes/tubercules & Manioc, Macabo \\
\hline Ce tableau présente juste la ration alimentaire quotidienne consommée \\
par les artisans de l'exploitation artisanale du sable Sanaga à Nkol'Ossananga.
\end{tabular}

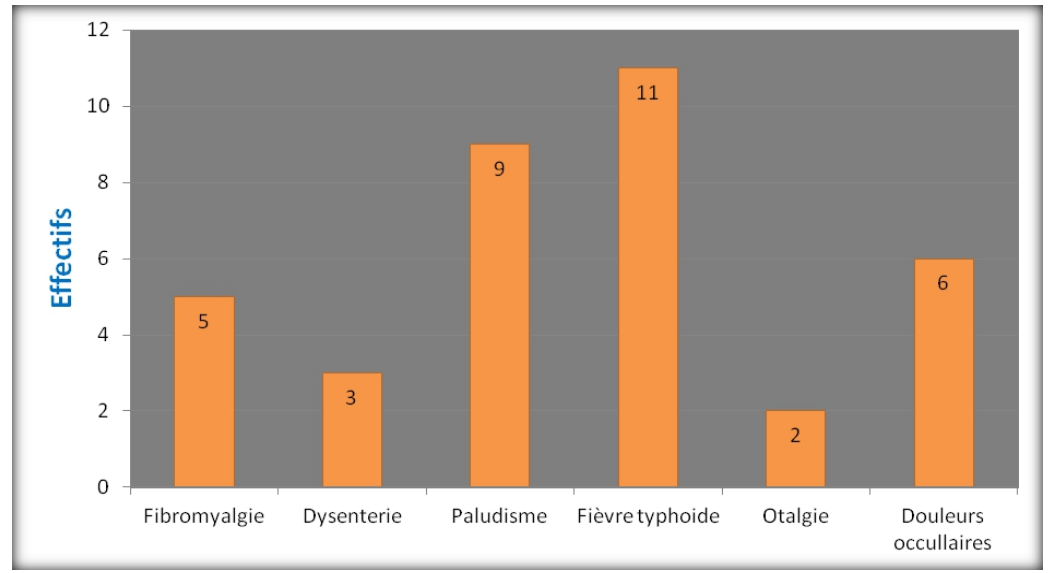

Figure 5. Etat de santé des artisans à Nkol'Ossananga. 


\section{Insécurité des artisans dans l'exploitation artisanale du sable à Nkol'Ossananga}

Pour exploiter le sable dans le lit du fleuve Sanaga, les artisans utilisent du matériel rudimentaire: pirogues (Figure 6a), seaux (Figure 6b) et pelles (Figure 6c). Sur le site, le sable se vend en camion, qui est l'unité de mesure. Pendant la mesure, les responsables de cette opération se disposent de façon à créer un espace très réduit entre eux, s'exposant ainsi à de nombreux accidents. Très souvent, ces artisans travaillent sans équipements de sécurité (chaussures de sécurité, gants, cache-nez, etc. pour les chargeurs ou palme, combinaison, etc. pour les plongeurs). Les plongeurs, pour partir de la surface jusqu'au fond sont en contact permanent avec l'eau du fleuve. Ne disposant pas d'équipements de protection individuelle (EPI), ils subissent les variations de pression et de température entre le fond du fleuve et la surface. Dans leurs mouvements répétitifs de plongées, ils bloquent instantanément leur respiration. Dans la plupart des cas, ils absorbent une quantité non négligeable d'eau du fleuve Sanaga. Le tableau II présente les résultats de l'analyse des échantillons d'eau du fleuve Sanaga au laboratoire de la Faculté d'Agronomie et des Sciences Agricoles (FASA).

Ce tableau montre que l'analyse des échantillons d'eau du fleuve Sanaga au laboratoire a porté essentiellement sur les caractéristiques cette dernière ( $\mathrm{pH}$, conductivité électriques, turbidimétrie) et sur sa composition chimique.

Les résultats de cette analyse montrent que les propriétés physiques de l'eau du fleuve Sanaga ne sont pas modifiées malgré l'exploitation poussée du sable de ce fleuve; seule la turbidimétrie a beaucoup augmenté, avec une valeur de 106,8 NTU, alors que la valeur standard est légèrement inférieure à 4 NTU. Cette valeur très élevée de la turbidité de l'eau du fleuve Sanaga est la conséquence directe des mouvements des particules de sables au fond du fleuve.

Quant aux résultats de l'analyse chimique, les éléments chimiques azote nitrique, magnésium et fer sont présents en excès dans l'eau du fleuve Sanaga. Globalement, l'eau Sanaga n'est pas une eau potable. Par conséquent, la consommation de cette eau, si minimale soit-elle, peut engendrer les risques énormes sur l'état de santé des artisans. Or, pendant leur mouvement de plongée, du fait du manque d'équipement de sécurité, les plongeurs boivent involontairement une fine quantité de cette eau, s'exposant dès lors aux maladies énormes. D'autre part, du fait du manque d'eau potable sur le site, les artisans se trouvent généralement confrontés au problème de soif intensive, les contraignant à consommer l'eau du fleuve Sanaga, malgré son niveau de pollution ou sa couleur désagréable. 


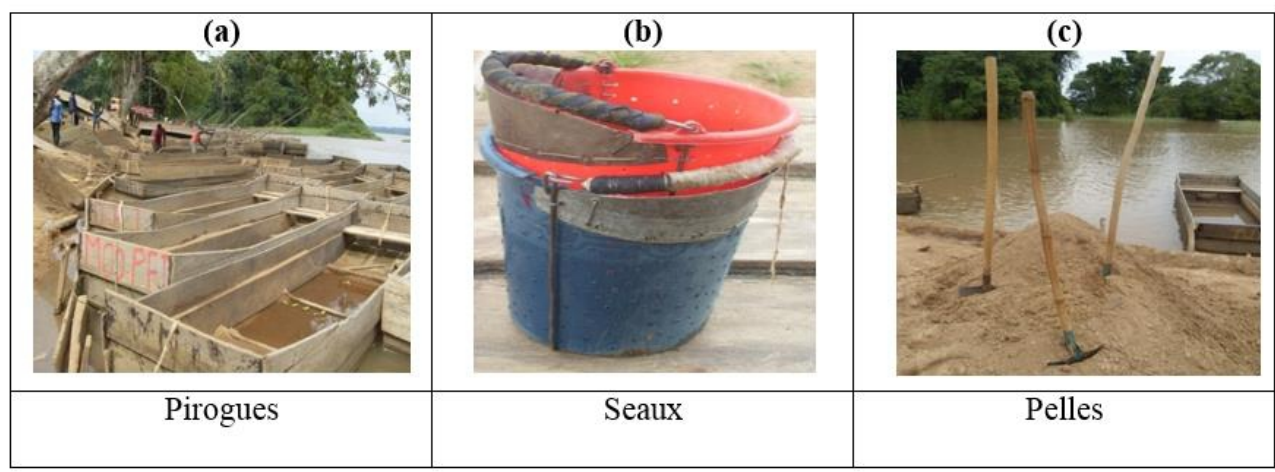

Figure 6. Matériel utilisé par les artisans dans l'exploitation du sable Sanaga à Nkol'Ossananga.

Tableau III. Résultats d'analyse des échantillons d'eau du fleuve Sanaga; ND : Non Défini. Source : (Ekengoue, 2015)

\begin{tabular}{cccc}
\hline $\mathrm{N}^{\circ}$ & CODE LABO & $\begin{array}{c}\text { VALEURS } \\
\text { OBTENUES }\end{array}$ & $\begin{array}{c}\text { VALEURS } \\
\text { LIMITES }\end{array}$ \\
\hline 1 & PH & 6,9 & $6,5-8,5$ \\
2 & Conduct.élect.mscm-1 & 0,05 & $<3$ \\
3 & Turbidimétrie NTU & 106,8 & $<4$ \\
4 & Bicarbonates (mg/l) & 34,16 & $\mathrm{ND}$ \\
5 & Azote ammoniacal (mg/l) & 9,8 & $2-8$ \\
6 & Azote nitrique (mg/l) & 210 & $50-80$ \\
7 & Calcium (mg/l) & 8,4 & $\mathrm{ND}$ \\
8 & Magnésium (mg/l) & 204,12 & $\leq 50$ \\
9 & Potassium (mg/l) & 0,523 & $\mathrm{ND}$ \\
10 & Sodium (mg/l) & 0,976 & $\mathrm{ND}$ \\
11 & Fer (mg/l) & 9,952 & $<1,5$ \\
\hline
\end{tabular}

\section{Impacts environnementaux de l'exploitation du sable}

Les activités accessoires à l'exploitation du sable Sanaga telles que la menuiserie (Figure 7a), la mécanique (dépannage, lubrification et nettoyage de moteurs des camions de transport du sable et tout autre véhicule entrant au site) et le petit commerce (vente de nourritures et des vêtements) génèrent d'énormes quantités de déchets. L'ensemble de ces déchets comprend le goudron, la sciure, les huiles de vidange et les déchets plastiques. Le premier, dérivé du pétrole brut, est utilisé sur le site pour la fabrication des pirogues. Chaque jour, en moyenne dix (10) litres de goudrons sont utilisés à Nkol'Ossananga, soit pour renforcer la capacité des vielles pirogues, soit pour en fabriquer de nouvelles. La cuisson du goudron pour la fabrication des pirogues met en jeux deux impacts environnementaux : d'abord la pollution de l'atmosphère suite à la libération dans l'air (Figure 7b) des produits toxiques comme le soufre et le chlore provenant de l'évaporation de l'huile , et ensuite la pollution du sol par le déversement de ce goudron sur celui-ci 
(Figure 7c). La pollution de l'air s'effectue aussi par la poussière qui s'élève lors de l'entrée des camions au site.

La présence des huiles de vidange dans la sablière de Nkol'Ossananga a pour origine l'activité mécanique. Cette activité est celle liée au dépannage des camions de transport en panne sur le site. Le plus souvent, lorsqu'il ne s'agit pas de changer le pneu d'un camion de transport, il est question de démonter tout le moteur du camion en panne, de le dépanner, puis de le remonter à nouveau. Ces différentes opérations (démontage, dépannage et remontage du moteur de camions) sont la source du déversement des huiles moteurs sur le sol dans la carrière de sable de Nkol'Ossananga. Par contre, les déchets plastiques proviennent de l'activité commerciale. Ces déchets (goudron, huiles de vidange/lubrifiantes, déchets plastiques) sont constitués de composés chimiques tels que les hydrocarbures aromatiques polycycliques (HAP), le soufre (S), les polychlorobiphényles (PCB), le tributyletain (TBT), le dichlorodiphenyltrichloroethane (DDT), le plomb $(\mathrm{Pb})$, le cadmium $(\mathrm{Cd})$, agents de pollution des différents milieux de l'écosystème (Commission Européenne, 2006).

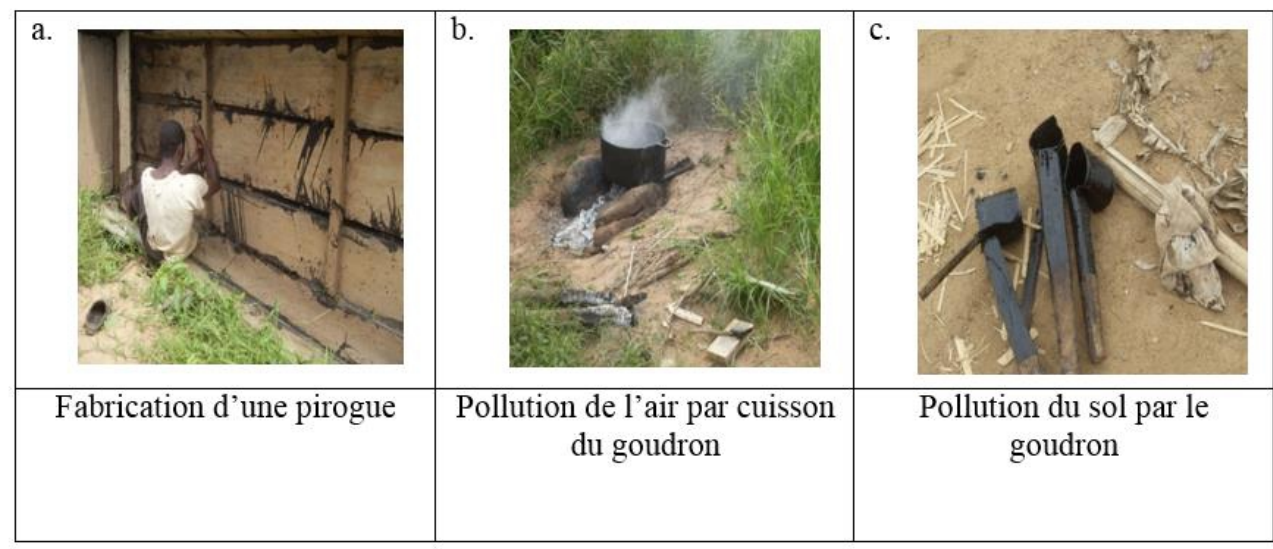

Figure 7. Impacts environnementaux de l'exploitation artisanale de sable à Nkol'Ossananga.

Le bois est utilisé à Nkol'Ossananga soit comme source d'énergie, soit comme matériel de fabrication des pirogues. En tant que combustible, il libère lors de sa combustion le monoxyde et le dioxyde de carbone $\left(\mathrm{CO}\right.$ et $\left.\mathrm{CO}_{2}\right)$ qui sont des gaz à effet de serre (Abanda et al., 2014). Cette utilisation du bois comme source d'énergie, mais aussi comme matière première dans la fabrication des pirogues est la cause de la pression que subit la végétation à Nkol'Ossananga. Sur le site d'exploitation, on dénombre des centaines de pirogues construites à partir du bois. Des enquêtes menées montrent qu'une pirogue est fabriquée avec environ dix (10) planches et trois (3) lattes .Qu'il s'agisse des planches ou des lattes, la longueur reste la même; $5 \mathrm{~m}$. En revanche, les planches ont une largeur variant entre 25 et $30 \mathrm{~cm}$ tandis que les 
lattes ont une largeur ne dépassant pas $10 \mathrm{~cm}$ (voir Figure 8 ci-dessous). Par ailleurs, le plus gros arbre de la région produit généralement cinquante (50) planches, le moyen arbre produit trente (30) planches et le plus petit arbre produisant jusqu'à dix (10) planches. Dans la localité de Nol'Ossananga, au regard du climat de la région (climat équatorial tropical chaud humide), les arbres sont généralement d' hauteurs très élevées (Figure 9). Ainsi, les petits arbres abattus par les scieurs pour les planches de fabrication des pirogues ont une taille pouvant atteindre $15 \mathrm{~m}$, les moyens $20 \mathrm{~m}$ et les gros arbres $30 \mathrm{~m}$.

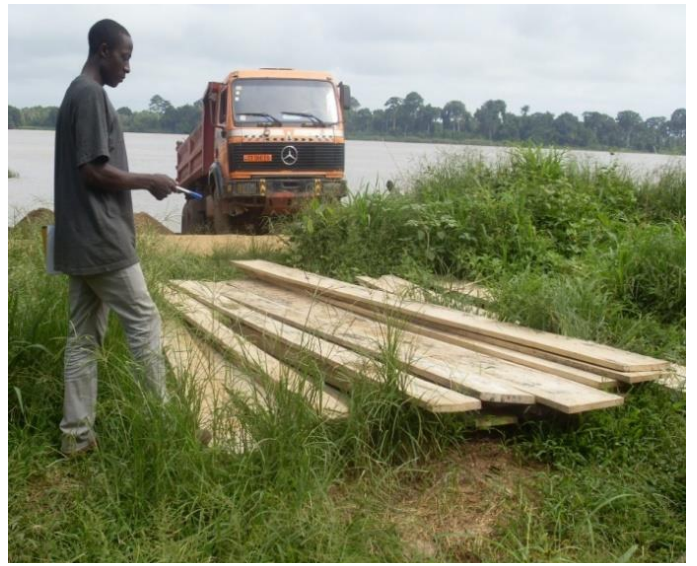

Figure 8. Planches et lattes utilisées pour la fabrication des pirogues dans la carrière artisanale de sable de Nkol'Ossananga

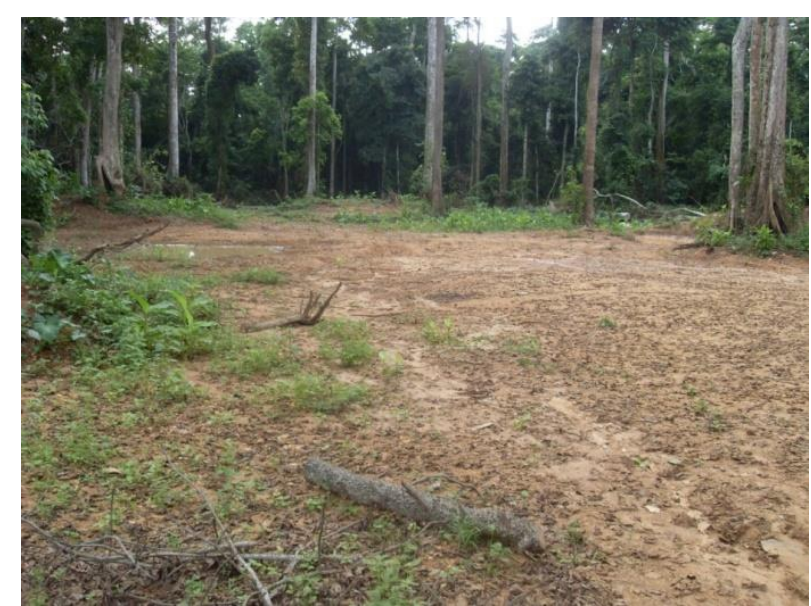

Figure 9. Forêt d'arbres de la localité de Nkol'Ossananga

Le taux de productivité mensuel des pirogues varie en fonction des saisons. Il peut atteindre dix (10) pirogues pendant la saison de crue où la production est faible et aller jusqu'à quarante (40) pirogues pendant la période d'étiage où la production est élevée. Cette affirmation vient du fait que :

* Pendant la période de crue, période allant généralement du mois de Mai au mois d'Octobre, le niveau des eaux du fleuve Sanaga augmente 
fortement (Figure 10a), ce qui constitue un danger énorme pour les plongeurs qui cessent de se rendre en plein fleuve pour faire des plongées. Seuls les plus courageux continuent à les effectuer en vue de ramasser le sable au fond du fleuve. L'activité d'exploitation artisanale du sable du fleuve Sanaga tourne ainsi au ralenti et le prix de vente d'un camion de sable augmente, allant de 60.000 FCFA à 120.000 FCFA. L'abandon de l'activité de plongée par plusieurs plongeurs demeure ainsi la cause de la baisse de la production des pirogues utilisées sur le site.

* Pendant la période d'étiage par contre, période allant du Mois de Novembre au Mois d'Avril, le niveau des eaux baisse (Figure 10b), et tous les plongeurs reprennent leur activité de plongée. La demande de pirogue augmente et la production du sable augmente, ce qui conduit à la baisse du prix du camion de sable qui peut aller jusqu'à 45.000 FCFA.

Comme conséquence de l'augmentation de la production de pirogues dans la dernière période, on assiste à un taux non négligeable de déforestation engendré par l'exploitation du sable du Sanaga à Nkol'Ossananga. A ces impacts sur l'environnement, s'ajoute l'agrandissement du lit du fleuve Sanaga, suite à l'érosion que subit le talus.

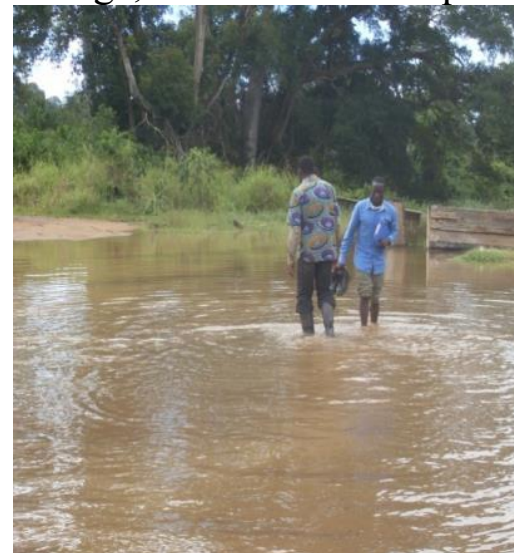

(a)

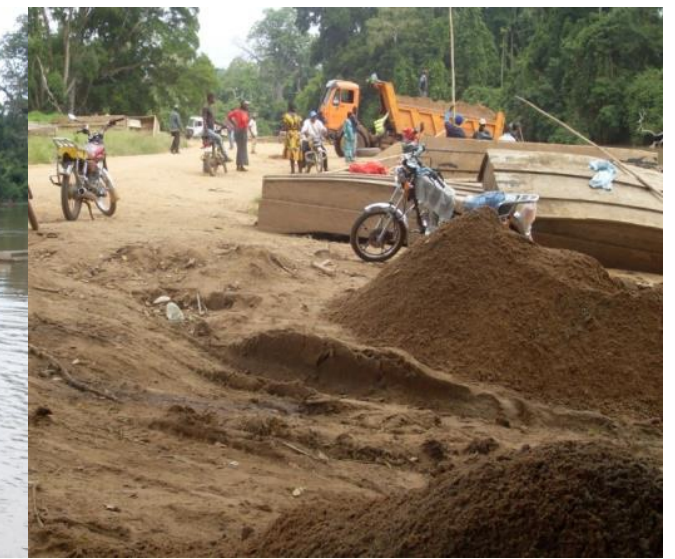

(b)

Figure 10. Exploitation du sable Sanaga à Nkol'Ossananga : (a) abandon de l'activité par les artisans du fait de l'augmentation du niveau des eaux du Sanaga ; (b) reprise des activités du fait de la baisse du niveau des eaux Sanaga.

\section{Discussion}

Le régime alimentaire pauvre, très déséquilibré au potentiel rénal acide (Remer et al., 1995) et très élevé (Tableau IV) auquel sont exposés les artisans, peut aboutir à l'acidification de leur organisme (Abanda et al., 2014). La légère augmentation du potentiel rénal acide peut entraîner la diminution extracellulaire du bicarbonate, pouvant générer à long terme des complications pour le développement et l'évolution de plusieurs conditions pathologiques (Mayerling et al., 2015). Plusieurs autres effets produits par le déséquilibre 
acido-basique, tels que la diminution progressive et graduelle du contenu minéralogique osseux, le retard de croissance chez les enfants, les risques d'ostéoporose et de sarcopenia chez les adultes et les personnes agées, l'hypercalciuria et la formation du calcul rénal, conséquence directe de l'acidosis méthabolique infraclinique (Fressetto et al., 2001 ; Reddy et al., 2002 ; Arnett, 2008 ; Ute et al., 2005; Welch et al., 2013) peuvent être observés. Au regard des résultats obtenus au laboratoire d'analyse de la FASA, la consommation de petites quantités d'eau du fleuve Sanaga par les plongeurs pendant leur activité, les expose aux maladies comme des lésions du foie, du cœur, du pancréas, des glandes endocrines et des articulations engendrées par l'accumulation du fer dans l'organisme. Une eau très concentrée en molécules d'azote est inappropriée pour la consommation humaine, notamment lorsque sa concentration dépasse le seuil $(50 \mathrm{mg} / \mathrm{l})$ fixé par l'Organisation Mondiale de la Santé (Obeidat et al., 2007). La consommation d'une eau riche en nitrate expose aux maladies telles que les cancers de la vessie (Hui-Fen et al., 2007) de l'estomac (Sandor et al., 2001), de l'œsophage et du duodénum (Ward et al., 2008 ; Khademikia et al., 2013). Plusieurs études de même ordre (impacts de l'exploitation artisanale du sable sur la santé, la sécurité et l'environnement) sous le thème 'impacts sociaux économiques de l'activité d'exploitation artisanale du sable ont été menées dans le monde. Il s'agit de: Adjoussi, 2001 ; Kombieni, 2016 ; Affagnon \& Honfoga, 2005 ; Donou, 2007 ; Atchegbe, 2015 ; Ngameni et al., 2017 ; Manga et al., 2013). Quant aux autres éléments présents en quantité élevée dans l'eau du fleuve Sanaga (Magnésium, Calcium, Sodium, Potassium, Bicarbonates), leur consommation et accumulation dans l'organisme des artisans (particulièrement les plongeurs) peut entraîner des symptômes particulièrement importants pouvant aboutir aux effets toxiques se traduisant en une confusion mentale et des troubles cardiaques.

Sur le plan sécuritaire, très souvent, le chargement d'un camion s'effectue simultanément au déchargement des pirogues. Ces deux activités menées au même moment constituent un risque énorme, pouvant aboutir à une perte de vie surtout pour les déchargeurs. D'autre part, l'absence des éléments de première nécessité (dortoir, restaurant, boite à pharmacie pour les premiers soins, toilettes pour éviter des défécations à l'air libre, garage et menuiserie) observé sur le site d'exploitation artisanale de Nkol'Ossananga, suscite une complète insalubrité du site d'une part et le taux élevé d'exposition des artisans à de nombreuses maladies d'autre part. 
Tableau IV: Nombre d'arbres coupés par catégorie et par saison pour la fabrication des pirogues

\begin{tabular}{|c|c|c|}
\hline Catégorie d'arbres & Période de crue & Période d'étiage \\
\hline Petits arbres & 10 & 30 \\
\hline Arbres moyens & 4 & 10 \\
\hline Grands arbres & 2 & 6 \\
\hline Moy/mois & 5 & \\
\hline Moy/an & & 130 arbres \\
\hline
\end{tabular}

Tableau V. Regroupement par classe des aliments couramment consommés dans la carrière de sable de Nkol'Ossananga. L'indice PRAL (Potential Renal Acid Load ou Charge Rénale

Acide Potentielle) évalue l'acidité de l'urine. Il additionne tous les minéraux acides, et soustrait tous les minéraux basiques.

\begin{tabular}{|c|c|c|}
\hline Classe d'aliments & Aliments & Indice PRAL (méq/100gr) \\
\hline Matières grasses & Beurre & 0,6 \\
\cline { 2 - 3 } & Huile de palme & $/$ \\
\hline Céréales & Riz brun & 12,5 \\
& Riz blanc & 4,6 \\
\hline Viande/Poisson & Pain & 1,8 \\
\hline Viande de bœuf & 7,8 \\
\hline Legumes/tubercules & Poisson & $/$ \\
\hline bsrcccc & Manioc & $/$ \\
\hline Boissons & Macabo & $/$ \\
& Bière & 0,9 \\
\hline & Jus de citron & $-2,5$ \\
\hline & Jus orange & $-2,9$ \\
\hline & Coca-cola & 0,4 \\
\hline & Vin blanc & $-1,2$ \\
\hline \multicolumn{2}{|c|}{ Total PRAL alimentation } & $22 \mathrm{mEq} / 100 \mathrm{gr}$. \\
\hline
\end{tabular}

La forte pollution du milieu a lieu à cause de ces facteurs:

- $\quad$ Les déchets provenant de l'activité du commerce due au petit marché initié sur le site d'exploitation du sable Sanaga à Nkol'Ossananga (Figure 11). - $\quad$ L'évaporation et le déversement du goudron dans l'air et sur le sol lors de la sa cuisson et son utilisation pour la fabrication des pirogues respectivement (Figure 12).

- $\quad$ L'absence des toilettes, poussant les artisans et toutes personnes sur le site à évacuer n'importe où, aux alentours du site en exploitation (Figure 13). Par conséquent, sur le site, apparaissent très souvent des odeurs toxiques caractéristiques de la pollution de l'air. Sur ces tas de selles se déposent des mouches qui par la suite sillonnent les plats de nourritures que consomment 
les artisans. Ceci est un danger énorme sur l'état de santé des artisans ou toute personne entrant dans le site.

- La pollution de l'air est aussi provoquée par les poussières qui se lèvent lors de l'entrée d'un camion dans le site.

- Globalement, sur le site, on observe un désordre total sur le mode de vie des artisans. Un régime alimentaire non varié est observable en raison de l'abandon de l'agriculture par les jeunes villageois au profit de l'activité d'exploitation du sable. Egalement, l'absence d'eau potable pour la consommation est remarquable sur le site.

Les huiles de vidange et le goudron déversés au sol et drainés dans les eaux de surface commencent par former une pellicule sur la surface. Les études de laboratoire de Surprenant et al. (1983), ont montré que des composés organiques volatils, semi-volatils et certains métaux qui constituent ces dérivés de pétrole passent de la phase huileuse à la phase aqueuse dans les eaux. Dans cette phase, certains métaux comme le zinc, le cuivre et le cadmium se trouvent sous forme dissoute et peuvent facilement s'infiltrer dans le sol tout en favorisant sa dégradation. Certains de ces composés organiques dont la tension de vapeur est élevée se volatilisent dans l'atmosphère et contribuent à la pollution de l'air ; tandis que les composés semi-volatils et les métaux se répartissent entre les phases eau et sédiment. Les huiles de vidange et le goudron peuvent aussi se dégrader par les processus d'altération, de coulage, d'émulsification, d'agglomération, de photo dégradation et la biodégradation (Payne et al., 1985). De façon générale, les huiles de vidange déversées sur le sol migrent en profondeur par gravité et peuvent atteindre les eaux souterraines puis se répandre latéralement sous l'action des forces capillaires et de l'hétérogénéité du sol (Stephens et al., 1981). En revanche, la déforestation poussée engendrée par la coupe excessive du bois à Nkol'Ossananga pour la fabrication des pirogues, conduit à la libération dans l'air du carbone fixé par les feuilles vivantes des arbres. Cette libération du dioxyde de carbone fixé par les plantes par photosynthèse contribue ainsi à l'augmentation de l'effet de serre (Rossi et al., 2015; Amiotte-Suchet, 2013). La conséquence à moyen terme est un changement climatique qui est au centre des préoccupations de la communauté internationale depuis des décennies. 

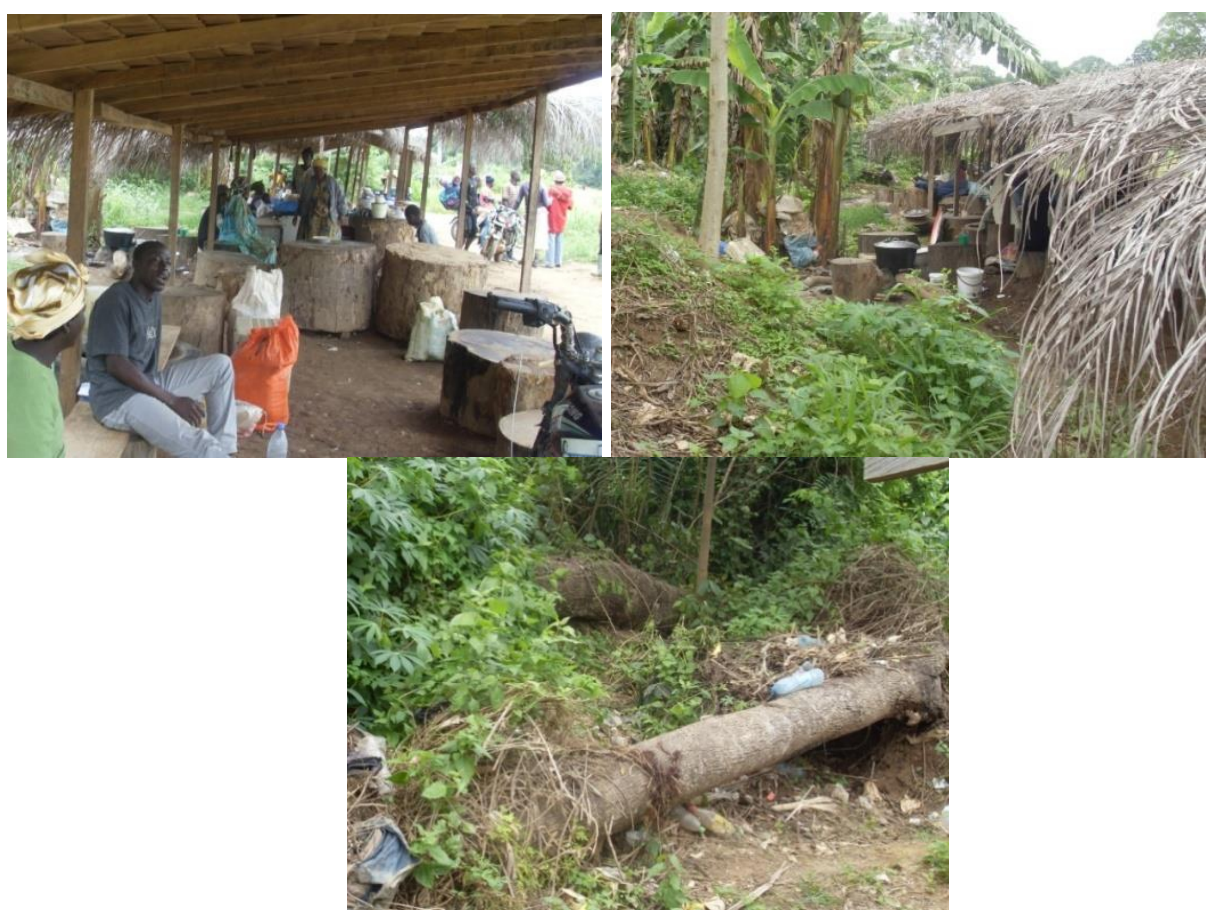

Figure 11. Cantine de vente de nourriture sur le site d'exploitation artisanale du sable de Nkol'Ossananga et déchets générés, contribuant à la forte pollution du milieu.

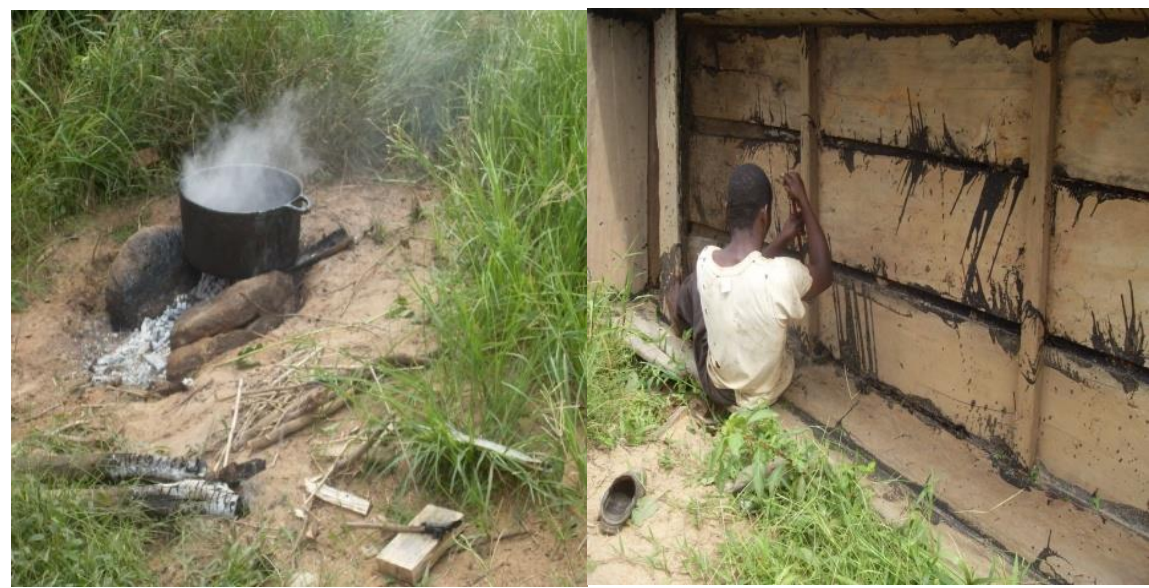

Figure 12. Evaporation du goudron dans l'air lors de sa cuisson et déversement au sol lors de son utilisation comme liant pendant la fabrication des pirogues. 


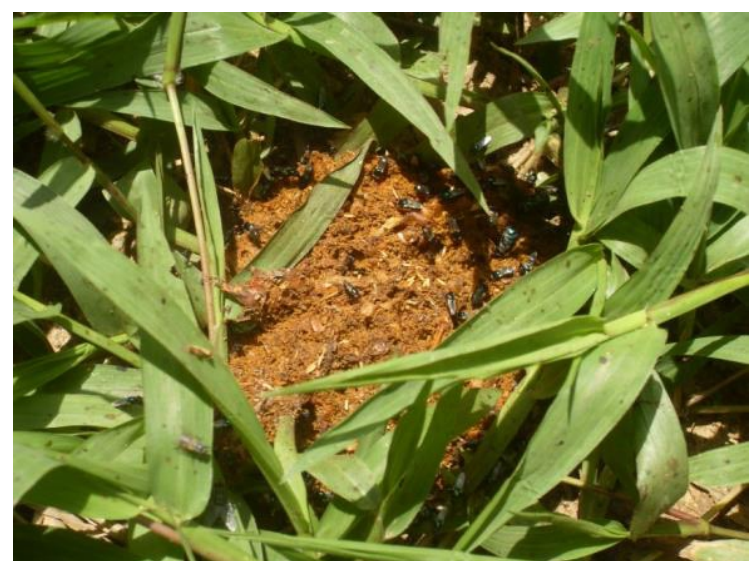

Figure 13. Tas de selles déposées à proximité du lieu de vente de nourritures sur le site d'exploitation du sable Sanaga.

L'amélioration des conditions sociales (pour les artisans) et environnementales n'est possible que via l'aménagement du site. En effet, au regard des nouvelles technologies de développement durable, il est possible de mettre en place un système de gestion des déchets générés par le petit marché sur le site, de façon non seulement à réduire le taux de pollution des sols mais aussi à produire soit de l'énergie ou du compost pouvant être utilisé dans l'agriculture. Il existe ainsi la possibilité de mettre en place à proximité $\mathrm{du}$ site d'exploitation une zone agricole afin de cultiver des aliments directement consommables sur le site par les artisans.

La construction des toilettes réduirait quant à elle le taux de pollution de l'air et du sol; car les artisans ne pourront plus évacuer n'importe où sur le site. En outre, la mise en place des salles dédiées à la construction des pirogues (salle de menuiserie) et d'entretien ou dépannage de camions entrant dans le site (salle de mécanique ou garage), réduirait elle aussi les impacts environnementaux de l'exploitation du sable du fleuve Sanaga , car les déchets seront collectés et canalisés pour un traitement approprié.

La construction d'un dortoir pourrait réduire au maximum les risques que courent très souvent les artisans en journée, se couchant sous des arbres et dans de vielles pirogues abandonnées (Figure 14), lorsqu'ils éprouvent le désir de se reposer.

De façon générale, les infrastructures sollicitées ont pour but majeur de réduire aux maximum les impacts sociaux et environnementaux liés à l'exploitation artisanale du sable Sanaga à Nkol'Ossananga. Dans cet ordre d'idées, une exploitation mécanisée est envisageable ; ce qui peut aussi avoir pour conséquence la production en quantité suffisante qui puisse satisfaire à cout sure la demande. Ainsi, la Figure 15 illustre un modèle de bâtiment approprié pour l'aménagement de carrière artisanale du sable Sanaga à Nkol'Ossananga. Cette figure présente une exploitation bien organisée avec 
des entrées et des sorties de camions bien ordonnées. Par ailleurs, le port des équipements de sécurité (casque, chaussures de sécurité, gants, etc.) limiterait les dangers encourus par les artisans.

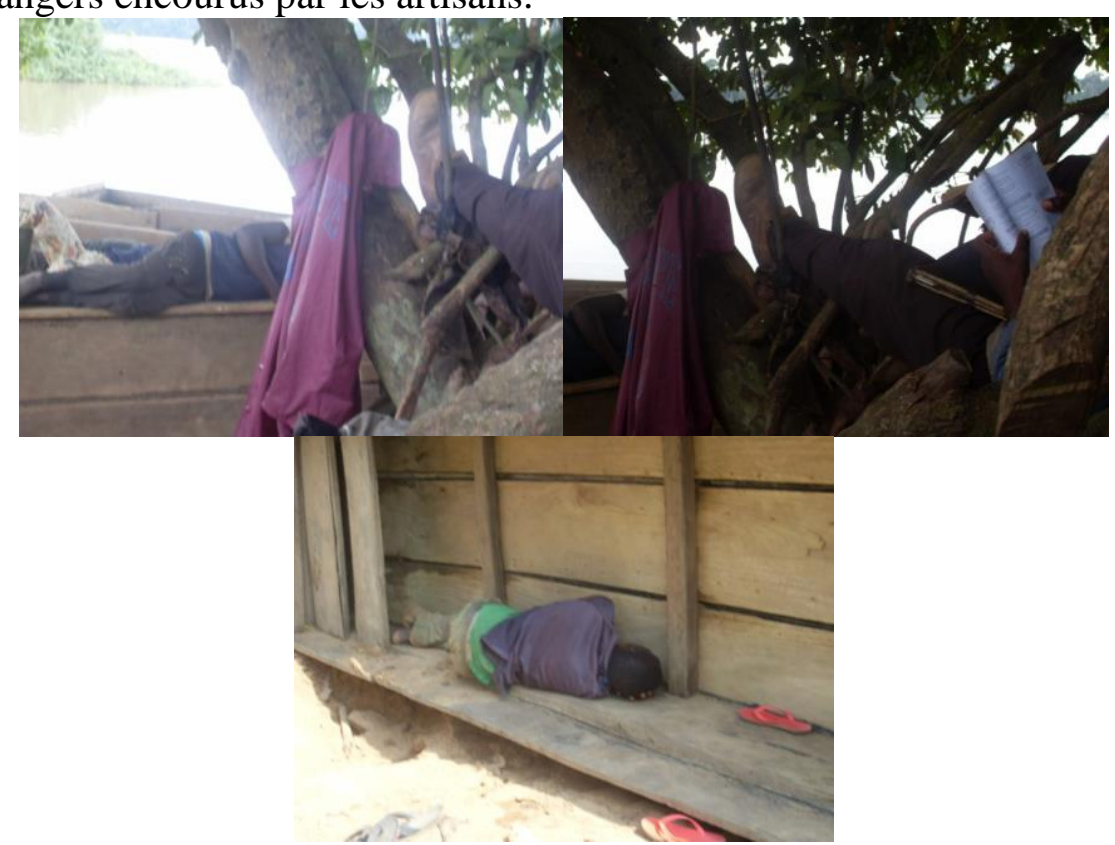

Figure 14. Artisans se reposants sous des arbres et sur des vielles pirogues abandonnées

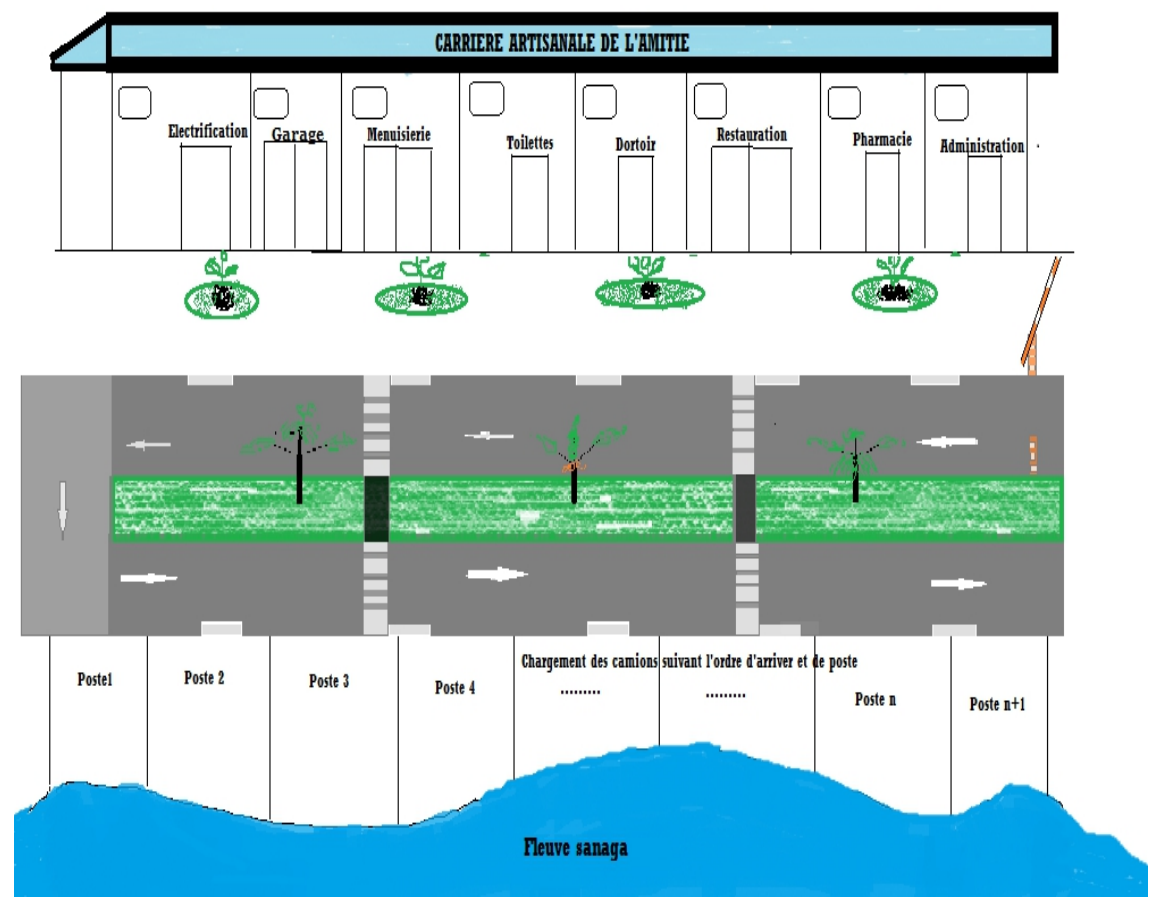

Figure 15. Model de construction appropriée pour l'aménagement de la carrière d'exploitation artisanale du sable Sanaga. 


\section{Conclusion}

L'exploitation artisanale de sable à Nkol'Ossananga laisse apparaitre une structure organisée en classes hiérarchisées. Cette organisation en différentes classes sociales fait de ce secteur d'activité un secteur important de l'économie nationale, contribuant à la survie des populations de plus en plus nombreuses. Mais, malgré cette importance, force est de reconnaître que ce secteur d'activité n'a pas encore atteint le développement escompté. En effet, au manque de moyens techniques et du travail pénible, s'ajoute l'insécurité pour les artisans, la population avoisinante et l'environnement en général. Pour une exploitation durable, des initiatives doivent être mises sur pied dans la carrière d'exploitation artisanale du sable Sanaga. Il s'agit de la mise en application des lois qui régissent l'exploitation artisanale au niveau des pouvoir publics, l'aménagement du site (construction des toilettes, de la pharmacie, du restaurant, du dortoir, etc.) pour les promoteurs de l'activité d'exploitation de carrières et l'utilisation des EPI chez les artisans. En outre, plusieurs autres travaux de recherche méritent d'être menés dans la carrière de sable de Nkol'Ossananga afin de compléter cette étude. Parmi celles-ci, il convient de citer l'estimation de la ressource en sable dans le lit du fleuve Sanaga ainsi que l'impact socioéconomique de l'exploitation du sable Sanaga à Nkol'Ossananga.

\section{Remerciements}

Une telle étude n'aurait pas pu être réalisée sans le soutien scientifique, technique et financier de nombreuses personnes et institutions. Ainsi, nous tenons à exprimer nos remerciements aux membres de l'association Green Heart International (GHI) qui ont travaillé avec nous tout au long de cette étude, à l'ensemble du corps enseignant de l'Université de Dschang, et plus particulièrement de la faculté des Sciences, à $\mathrm{M}$. Tcheumenack Kouémo Jules pour ses lectures minutieuses, à $\mathrm{M}$. Tébong Christophe pour son soutien dans la réalisation de la cartographie de la zone d'étude : Nous remercions aussi $\mathrm{M}$. Benoit Messanga pour l'accueil chaleureux dans la carrière de Nkol'Ossananga et son soutien financier, ainsi que l'ensemble des artisans de Nkol'Ossananga pour leur sympathie et collaboration. Enfin, nos remerciements à tous ceux qui ont contribué à la réalisation de cette étude.

\section{References:}

1. Abanda, F.H., Manjia, M.B., Cole, E., \& Mempouo. B. (2014). The Potential of Efficient Mud-bricks Cookstove in Cameroon: An Exploratory Study. Macrothink institute TM. Doi : 10.5296/emsd.v4il.6715.

2. Amiotte-Suchet, P. (2013). Le cycle biogéochimique du Carbone. L'actualité chimique 371-372 : 25-29. 
3. Armett, T.R. (2008). Extracellular $\mathrm{pH}$ regulates bone cell function. J. Nutr 138(2): 415S-418S.

4. Billon, P.L. (2001). The political ecology of war: natural resources and armed conflicts. The Journal of Political Geography 20 (5): 561-584.

5. Collier, P. (2000). Rebellion as a quasi-criminal activity. The journal of conflicts resolution 44 (6): 839-853.

6. Commission Europenne (2006). Traitement des déchets. Edificio Expo, Inca Garcilaso s/n, E-41092 Séville, Espagne.

7. Ekengoue, M. C. (2015). Santé et sécurité dans l'exploitation minière artisanale : cas de la carrière artisanale de sable de Nkol'Ossananga, Centre-Cameroun. Mémoire de Master professionnel, Département des Sciences de la Terre, Université de Dschang, 73p.

8. Fressetto, L.A., Morris, R.C., Sellmeyer, D.E., Todd, K., \& Sebastian, A.D. (2001). Evolution and againg. The patholophysiologic effects of the post agricultural inversion of the potassium to sodium and base-tochloride ratios in the human diet. Eur.J.Nutr 40(5): 200-213.

9. GADHOP (Groupe d'Association de Défense des droits de l'Homme et dela Paix) (2012). Etude socioéconomique sur l'exploitation minière artisanale dans le territoire de Lubero, régions de Kasugho, Katanga/Buyinga et Manguredjipa. Ed Ghadiop. Impression aux Générales des imprimeries de Butembo.

10. Geenen, S. \& Custers, R. (2010). Tiraillements autour du secteur minier de l'Est de la RDC. L'Afrique des grands lacs : Annuaire. ISSN : 1636-208X

11. Goh, D. (2016). L'exploitation artisanale de l'or en côte d'ivoire : la persistance d'une activité illégale. European scientific journal. Doi : 10.19044/esj.2016.v12n3p18.

12. Hui-Fen, C., Shang-Shyue, T., \& Chun-Yuh, Y. (2007). Nitrate in drinking water and risk of death from bladder cancer: an ecological case-control study in Taiwan. Journal of toxicology and environmental Health, Part A : 70 (20) : 1000-1004.

13. Kemgang, Y. L. D. (2015). Evaluation de la pollution de l'eau due à l'activité de la sablière de Tsoutsang en vue de proposer des mesures d'atténuation. Mémoire de Master professionnel, Département des Sciences de la Terre, Université de Dschang, 54p.

14. Khademikia, K., Rafiee, Z., Mehdi, Amin M., Poursafa, P., Mansourian, M., Modaberi, A. (2013). Association of nitrate, nitrite and total organic carbon (TOC) in drinking water and gastrointestinal diseases. Journal of Environmental and public Health, 1-5. http://dx.doi.org/10.1155/2013/603468.

15. Kyamwami, P.K. (2013). Travail des enfants dans le site minier d'exploitation artisanale de Bisie en territoire de Walikale. Une crise 
oubliée en République démocratique du Congo. IPIS publications, Rapport d'étude évolutive 2007-2012, 36p.

16. Mayerling, L.S., Bemal, J., \& Lopez, M. (2015). Dietary potential renal acid load in Venezuelan chidren. Nutr Hosp 31(5): 2054-2061.

17. Midende, G. (2010). Les exploitations minières artisanales du Burundi. L'Afrique des grands lacs : Annuaire. ISSN : 1636-208X.

18. Mutsima, C.K., Kindele, J.M., Tshimwanga, J.A., \& Kahadi, T.P.A. (2015). De l'exploitation minière artisanale et son impact environnement dans la ville de Kolwezi. KAS African Law Study Library. Doi : 10.5771/2363-6262-2015-3-581.

19. Obeidat, M.M., Massadeh, A.M., Al-Ajlouni, A.M., \& Athamneh, F.S. (2007). Analysis and evaluation ofnitration levels in groundwater at Al-Hashimiya area, Jordan. Environmental Monitoring and Assesment 135, 1-3: 475-486.

20. Payne, J.R. \& Phillips, C.R. (1985). Photochemistry of petroleum in water. Environ. Sci. Technol. 19: 569-579.

21. Razack, A.A. (2012). Proposition pour l'optimisation de la mine artisanale au Niger. Proposals for optimizing artisanal mining in Niger. Pangea, HAL archives. HAL id: insu-00947881.

22. Reddy, S.T., Wang, C.Y., Sakhaee, K., Brinkley, L., \& Pack, C.Y. (2002). Effect of low-carbohydrate high-protein diet on acidbasebalance, stone forming propensity, and calcium metabolism. American Journal of Kidney Deseases 40(2): 265-274.

23. Remer, T. \& Mane, F. (1995). Potential rhenal acid load of foods and its influence on urine pH. J.AmDiet Assoc 95 (7):791-7.

24. Rossi, M., André, J., \& Vallauri, D. (2015). Le carbone forestier en mouvements. Eléments de réflexion pour une politique maximisant les atouts du bois. Lyon, rapport REFORA, 40p.

25. Sandor, J., Kiss, I., Farkas, O., \& Ember, I. (2001). Association between gastric cancer mortality and nitrate content of drinking water: ecological study on small area inequalities. Eur. J. Epidemiole 17 (5) : 443-7.

26. Sba-Ecosy-CEDRES (2011). Analyse économique du secteur des mines: liens pauvreté et environnement. Rapport final du projet d'Initiative Pauvreté et Environnement (IPE), préparé pour le Ministère de l'Environnement et du cadre de vie (MECV) du Burkina Faso, 69p.

27. Sinonda, M. (2010). Cours de l'administratif et développement. G3 droit public, Unikol/ Kolwezi, inédit.

28. Stephens, R.D., Hatayama, H.K., Holden, A.M., Brown, B.A., \& Raymond, G.E. (1981). A Study of the Fate of Selected Toxic Materials in Waste Oils Used for Dust Palliation on Logging Roads in 
the Plumas National Forest, submitted in Partial Fulfilment of U.S. Department of Agriculture (Forest Service), Contract No. 53-91S8-86132 for the Hazardous Materials Laboratory Section, California Department of Health Services, Berkeley, CA.

29. Surprenant, N.F., Battye, W.H., Fennelly, P.F., \& Brinkmann, D.W. (1983). The Fate of Hazardous and Nonhazardous Wastes in Used-Oil Disposal and Recycling. Prepared for the United States Department of Energy, Washington, D.C., Contract No. DE-AC19-81BC10375. In Santé Canada. Santé de l'environnement et du milieu du travail (1994). ISBN : 0-662-98896-5.

30. Ute, A., Remer, T., Manz, F., Neu, C.M., \& Schoenau, E. (2005). Long term protein intake and dietary potential renal acid are associated with bone modeling and remodeling at the proximal radius in the healthy children. Am. J. Clin. Nutr 82: 1107-1114.

31. Ward, M.H., Heineman, E.F., Markin, R.S., \& Weisenburger, D.D. (2008). Adenocarcinoma of the stomach and esophasus and drinking water and dietary sources of nitrate and nitrite. International Journal of occupational and Environmental Health 14 (3): 193-197.

32. Welch, A.A., Macgregor, A.J., Skinner, J., Spector, T.D., Moayyeri, A., \& Cassidy, A. (2013). A higher alkaline dietary load is associated with greater indexes of skeletal muscle mass in women. Osteoporos int 24 (6) 1899-1908. 